\title{
Longitudinal Patterns of Care for Patients with Posttraumatic Stress Disorder
}

\author{
David L. Ronis, ${ }^{1,2,6}$ Elizabeth W. Bates, ${ }^{1,2}$ Adam J. Garfein, ${ }^{3}$ \\ Barbara K. Buit, ${ }^{1}$ Spencer P. Falcon, ${ }^{2,4}$ and Israel Liberzon ${ }^{2,5}$
}

This study assessed patterns of mental health service use over time by patients with posttraumatic stress disorder (PTSD) - as compared with patients with schizophrenia and major depression - with emphasis on the persistence and episodic versus continuous nature of use. Data on utilization were extracted from Veterans Health Administration (VA) administrative data bases. Temporal patterns of use were categorized into intervals of inpatient, outpatient, and no use. PTSD patients used substantial amounts of mental health services, but averaged 2.2 nonuse intervals lasting more than 100 days each, implying that use was episodic. Use of mental health services by patients with PTSD is substantial, persistent, and quite episodic. To the extent that use of services reflects the course of the disorder, the results suggest that remissions are usually followed by relapse, and that absence of symptoms does not mean that the disorder has run its course.

KEY WORDS: longitudinal; PTSD; mental health services; schizophrenia; major depressive disorder.

Because posttraumatic stress disorder (PTSD), an anxiety disorder precipitated by exposure to a traumatic event, has only recently been accepted into the mental health nosology (American Psychiatric Association [APA], 1980), its course over time has not yet been extensively studied. The few

${ }^{1}$ Ann Arbor VA Health Services Research and Development Field Program, Ann Arbor, Michigan 48113.

${ }^{2}$ University of Michigan, Ann Arbor, Michigan 48109.

${ }^{3}$ DEVANE Corporation, formerly with VA.

${ }^{4} \mathrm{Blue}$ Cross/Blue Shield of Michigan, formerly with VA.

${ }^{5}$ Department of Veterans Affairs.

${ }^{6}$ To whom correspondence should be addressed at VA HSR\&D (152), P.O. Box 130170, Ann Arbor, Michigan 48113-0170. 
studies that have examined the course of PTSD over time have found that PTSD may have an uneven or episodic course, including repeated cycles of quiescence, intrusion, and numbing (Horowitz, 1986) and exacerbation of symptoms triggered by stimuli (including the anniversary date) that remind the person of the original traumatic event (Green, Lindy, \& Grace, 1985; Kinzie, 1986; Sack et al., 1993; Solomon, Kotler, Shalev, \& Lin, 1989). Information about the long term persistence of PTSD comes from retrospective studies that assessed persons long after exposure to extreme stressors (Green, Lindy, Grace, \& Leonard, 1992; Kulka et al., 1990a, 1990b; Weiss et al., 1992). The National Vietnam Veterans Readjustment Study (NVVRS) (Kulka et al., 1990a, 1990b) found that between 32 and $49 \%$ of Vietnam veterans who ever had PTSD still had it in 1986, more than 10 years after the end of the war. Similarly, Green et al. (1992) found that $42 \%$ of a sample of dam collapse victims who ever had PTSD had it 14 years after the disaster. If PTSD symptoms are more or less constantly present until some point after which they no longer occur, these findings suggest that PTSD has a half-life of about 10 years. If, on the other hand, symptoms of PTSD temporarily remit or fluctuate in magnitude over time, finding that a third to a half had diagnosable PTSD during a 1-year period many years after exposure may mean that the others were simply in temporary remission. With this scenario, PTSD may be almost $100 \%$ persistent.

The goal of this research was to determine how intensively, persistently, and continuously patients with PTSD used VA mental health services over several years. To put the patterns of use by PTSD patients into perspective, they are compared with those of patients with two other chronic mental disorders: schizophrenia and major depressive disorder. Schizophrenia and major depressive disorder were chosen as comparison conditions because they are serious chronic mental disorders whose course has been thoroughly studied. While both are quite persistent, they differ in the continuity of their symptoms. About half of schizophrenia patients in longitudinal studies have a stable level of disability for many years, while half have an undulating or varying level. Somewhat over $50 \%$ reach an improved level of functioning with only mild impairment (Bleuler, 1978; Fenton \& McGlashan, 1991; Harding, 1988; Harding, Brooks, Ashikaga, Strauss, \& Brier, 1987; Marengo, Harrow, Sands, \& Galway, 1991). Major depressive disorder tends to be an episodic condition with multiple recurrences. While around $20 \%$ of patients remain consistently depressed for years, most patients remit within a year of treatment and experience long periods with normal mood and functioning between depressive episodes. The chance of recurrence after remission of a major depressive episode is high, especially among patients who have already had more than one episode (Keller et al., 1992; Shea et al., 1992; Stephens \& McHugh, 1991; Thase, 1990; Wells, Burnam, Rogers, Hays, \& Camp, 1992). 
The purposes of this research were to (1) characterize PTSD patients' patterns of mental health service use over time, and (2) compare patterns of utilization among patients with PTSD with those of patients with schizophrenia and major depressive disorder. In particular, we wished to characterize the intensity, persistence, and episodic (versus continuous) nature of mental health service use. The study used a prospective longitudinal design to examine utilization over $43 / 4$ years.

\section{Method}

\section{Patient Sample}

Patients in this study were identified in a 1986 survey of Veterans Health Administration (VA) outpatient mental health programs that collected data on all 104,729 patients who received outpatient mental health services from VA from September 8 through October 3, 1986. Survey data were recorded by each mental health care provider. Patients were eligible for inclusion in this analysis if they had been diagnosed with only one of the three target disorders to better discriminate patterns of use associated with each disorder. The study sample of 1,000 PTSD patients consisted of all 37 females with PTSD seen during the survey period who were not diagnosed with schizophrenia or major depressive disorder together with a random sample of 963 males with PTSD who did not have either of the comparison diagnoses. Comparison patients with schizophrenia and major depressive disorder were individually matched to PTSD patients on gender, age, and geographic location of visit. Analyses of utilization were restricted to the 939 patients with PTSD, 923 patients with schizophrenia, and 907 patients with major depressive disorder who did not die during the study period, so utilization could be examined for equal periods (see Table 1).

The diagnoses used to classify and select the patients in this study were simply the clinical diagnoses reported by providers in the outpatient setting. Most frequently, the providers were psychiatrists, followed (in order) by social workers, nurses, and psychologists. Given the low number of diagnoses listed per patient in the survey $(M=1.5)$, and the low rate of major depressive disorder diagnoses among patients with PTSD (about $20 \%$ in the survey compared to $26 \%$ to $84 \%$ lifetime comorbidity in other studies, c.f., Keane \& Wolfe, 1990), it is likely that comorbidities were underreported and that the groups selected in the study were far from pure. The PTSD group in particular probably includes many patients who also have major depressive disorder, with or without symptoms at the time of the survey. The implications of these characteristics of the sample will be addressed in the discussion. 
Patients in the three groups were indistinguishable on age $(F(2$, $2766)<1, p>.5)$, gender $\left(\chi^{2}(2)<1, p>.5\right)$ and region of the country $\left(\chi^{2}(6)<1, p>.5\right)$, the matching variables (see Table 1). Most were in their 30 s or 40 s in 1986 , and the vast majority ( $96 \%$ ) were male. In 1986, the patients differed across diagnostic groups on highest level of adaptive functioning $(F(2$, $2737)=84.8, p<.001)$, number of psychiatric diagnoses $(F(2,2768)=99.7$, $p<.001)$, and presence of a substance abuse comorbidity $\left(\chi^{2}(2)=38.0\right.$, $p<.001)$. Level of adaptive functioning was rated on Axis V of DSM-III from 1 for "superior" to 7 for "grossly impaired" (APA, 1980). Patients with schizophrenia were significantly more impaired (5.0) than patients with PTSD (4.5). Patients with major depressive disorder were statistically significantly less impaired (4.4) than patients with PTSD, but this small difference was not clinically important. Patients with schizophrenia had fewer psychiatric diagnoses recorded during the 1986 outpatient mental health survey (1.5) than patients with PTSD (2.0) who did not differ significantly from patients with major depressive disorder (2.1). Patients with PTSD were more likely (20\%) to have a substance abuse comorbidity than patients with schizophrenia (12\%) but did not differ in this regard from patients with major depressive disorder (23\%).

\section{Utilization Classification System}

Standard measures characterizing health care utilization (e.g., number of outpatient visits and number of inpatient days per year) gloss over individuals' changing patterns of use over time, and, therefore, are not adequate for identifying episodic versus continuous utilization or other sequential temporal patterns. To allow analysis of our data, including over 200,000 outpatient mental health visits and over 5,000 psychiatric hospitalizations, we classified longitudinal patterns of utilization by (a) dividing a patient's complete history of utilization of VA mental health services into a sequence of mutually exclusive and exhaustive intervals of use, (b) distinguishing inpatient from outpatient utilization, (c) categorizing outpatient use by frequency of visits, and (d) identifying periods of non-use of VA mental health care.

The system was based on past research on episodes of illness, analysis of the distribution of intervals between successive VA outpatient visits, and knowledge about typical patterns of mental health service delivery. Our system differs from other classifications of mental health service episodes (Hombrook, Hurado, \& Johnson, 1985; Keeler, Manning, \& Wells, 1988; Kessler, Steinwachs, \& Hankin, 1980) in that we separate qualitatively different kinds of use (e.g., inpatient and outpatient), focus more on periods of nonuse, and allow longer intervals between contacts before concluding that an "episode" has ended. In this study, only care provided in a distinctly mental health setting 


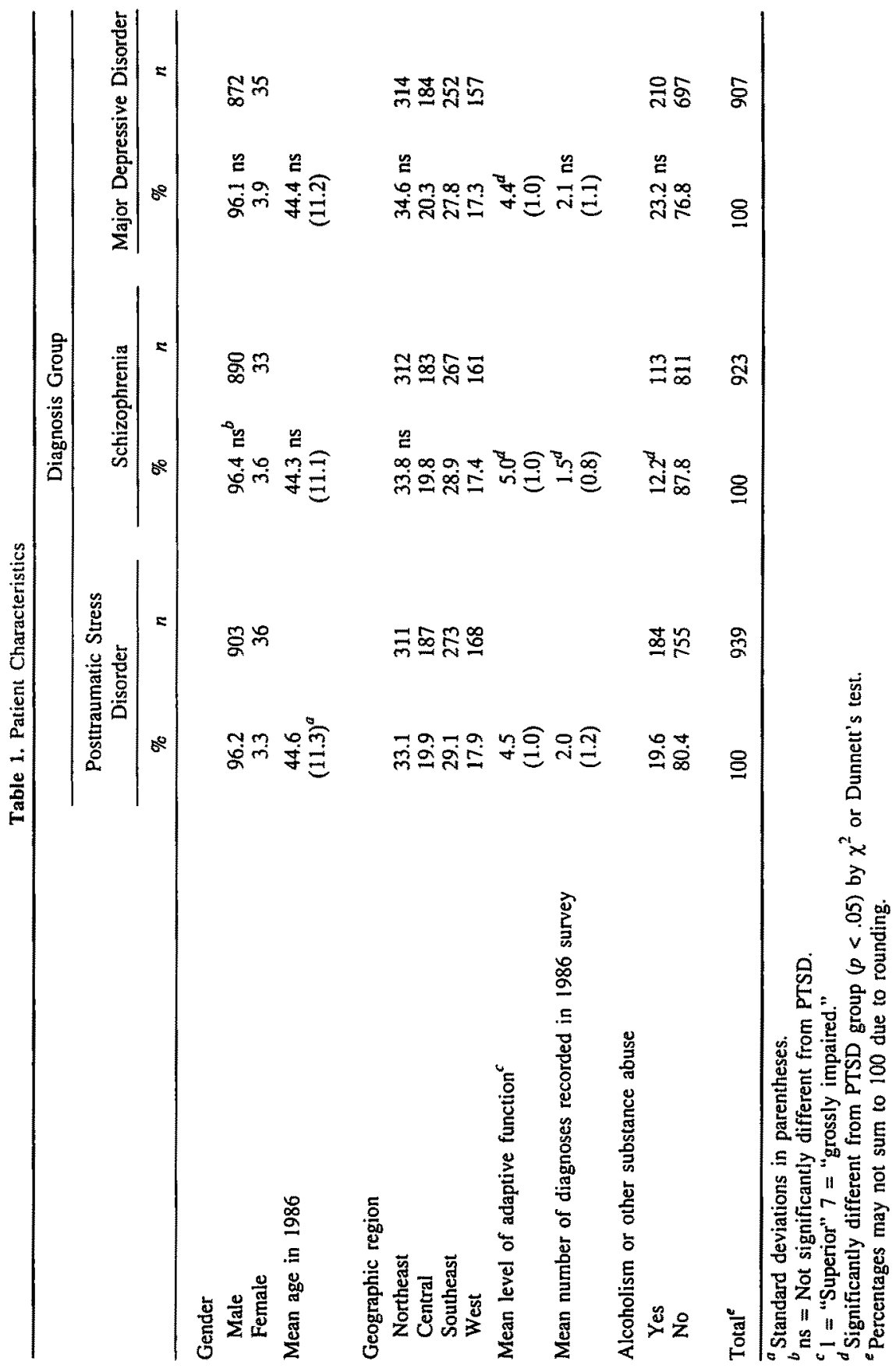


was measured. Although this restriction may underestimate mental health care by ignoring such care provided by generalists or in the context of care for physical conditions, the altemative approach of including all utilization would grossly overestimate mental health care. The categories and their definitions are presented below with some notes on the rationale for their selection.

An inpatient interval is any VA inpatient stay that includes time in a psychiatric (including substance abuse) unit. Time in nursing homes was not included because so little mental health care is provided in such settings (Burns, Wagner, \& Taube, 1993).

An active outpatient interval is any period of time characterized by one or more outpatient mental health visits, where all intervals between contacts with VA mental health care are less than or equal to 18 days. These intervals are intended to include visits up to 2 weeks apart, plus a few days latitude to allow for scheduling difficulties. This cut-off corresponds to a natural break in the distribution of inter-visit intervals. An outpatient maintenance interval is any period of time characterized by one or more outpatient mental health visits, where all intervals between contacts with VA mental health care are 19 to 65 days, inclusive. The cut off at 65 days corresponds to a natural break in the distribution of inter-visit intervals and is roughly consistent with previous 8-week episode boundaries (Keeler et al., 1988; Kessler et al., 1980). An outpatient low maintenance interval is any period of time characterized by one or more outpatient mental health visits, where all intervals between contacts are 66 to 100 days long, inclusive. This category was included so that there would be a clear distinction between steady outpatient utilization (categorized as active or maintenance) and nonuse.

A nonuse interval is any period of time greater than 100 days with no VA mental health contact. Past research defining episodes of psychiatric care (Keeler et al., 1988; Kessler et al., 1980) generally identified the end of an episode by a period of 8 weeks with no utilization. We chose 100 days rather than 8 weeks in light of the chronic nature of the conditions we were studying and the recognition that management of chronic mental illness (e.g., with neuroleptics) may sometimes permit visits as infrequently as every 3 months to assess and renew medication orders.

For completeness, two other kinds of intervals were defined. An infrequent outpatient interval is a day with a mental health outpatient visit surrounded by nonuse intervals. A short nonuse interval is any period of 100 days or less between two inpatient mental health stays in which there are no VA outpatient mental health visits. Even short periods without outpatient mental health visits between two inpatient stays are classified as nonuse since readmission without outpatient contact would generally be considered a failure of the delivery system. Because they accounted for so little of patients' time (less than $1 \%$ together), results for these two interval types are not reported. 
Example 1: Outpatient Use Only

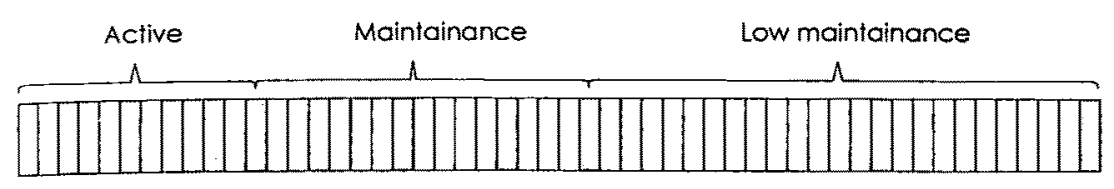

Example 2: Inpatient and Outpatient Use

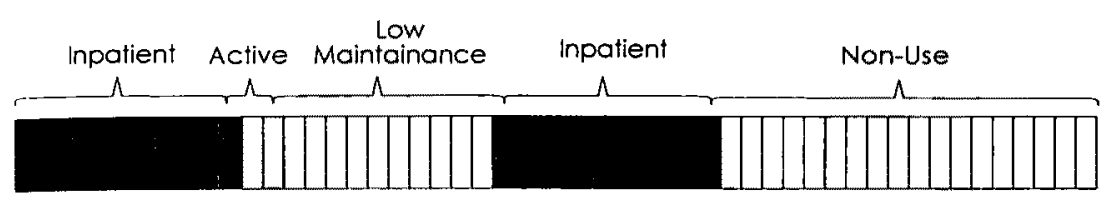

$\Pi$ No utilization $\square$ Outpatient Hospitalization

Figure 1. Classification of intervals: Hypothetical examples.

Examples of classification. Figure 1 illustrates this classification system. In order to fit a long time period and a variety of interval types into the figure, the week rather than the day has been used as the unit of time. At the beginning of Example 1, all visits are within 18 days of each other, so the first interval (about 12 weeks) is classified as active outpatient care. This is followed by many weeks classified as outpatient maintenance because visits are 19 to 65 days apart. The example concludes with almost half a year classified as low maintenance because visits are 66 to 100 days apart. Note that this system classifies time intervals rather than visits or days. For example, assuming there is a visit on 1 day in the 12th week that ends the active outpatient interval and begins the outpatient maintenance interval, that day is equally part of both intervals.

Example 2 shows two inpatient intervals. The time between the first two of these includes one outpatient visit in the second week. This visit is within 18 days of the hospital discharge, so the period from discharge to the visit is an active outpatient interval. The period from that outpatient visit to the next admission is between 66 and 100 days, so that period is classified as a low maintenance interval. There are no outpatient visits after the second inpatient stay. Since this period is more than 100 days long, it is classified as a nonuse interval. 
Period of observation. Data on all utilization of mental health care under VA auspices were pulled from VA data bases for the fiscal years 1986 to 1991: October 1,1985, to September 30, 1991. Classification of intervals at the beginning and end of any period of observation can be ambiguous, since the amount of time until the next or prior utilization is not known. So, utilization occurring before or during the 1986 outpatient survey (from which the patients were selected) and during the last 100 days in this period was used to permit unambiguous classification of all use from October 4, 1986, to June 22, 1991. This period of observation consisted of the $43 / 4$ years after the survey from which the patients were selected. By starting classification after the 1986 survey we could also be assured that the patients were diagnosed with PTSD, schizophrenia, or major depressive disorder prior to the utilization of interest. Each interval has a length of stay (LOS). Lengths of the first and last intervals in the window of observation are truncated at the beginning and ending dates of the window.

\section{Results}

PTSD Patients' Use of Mental Health Services: Intensity and Episodic Nature

The top panel in Table 2 uses the interval classification system to characterize patterns of use by PTSD patients. The percentage of time spent in each interval type is the best summary of each type of use because it incorporates information about the number of intervals and their lengths of stay. It is notable that PTSD patients spent more time (40\%) in nonuse intervals than in any other kind of interval.

Three out of four patients had at least one such interval. Most (56\%) had more than one (not shown in table). Across the entire group, patients had an average of 2.2 nonuse intervals. Patients who had at least one such interval had an average of 2.9 . The average duration of a nonuse interval was 314 days - more than 10 months.

After nonuse, outpatient intervals accounted for the largest amount of patients' time: active (22\% of time), maintenance (27\%), and low maintenance $(9 \%)$ intervals. Active intervals (90\% of patients; averaging 7.2 intervals per patient) and maintenance intervals (92\% of patients; also 7.2 per patient) were much more common than low maintenance intervals ( $61 \%$ of patients; 1.5 per patient). 


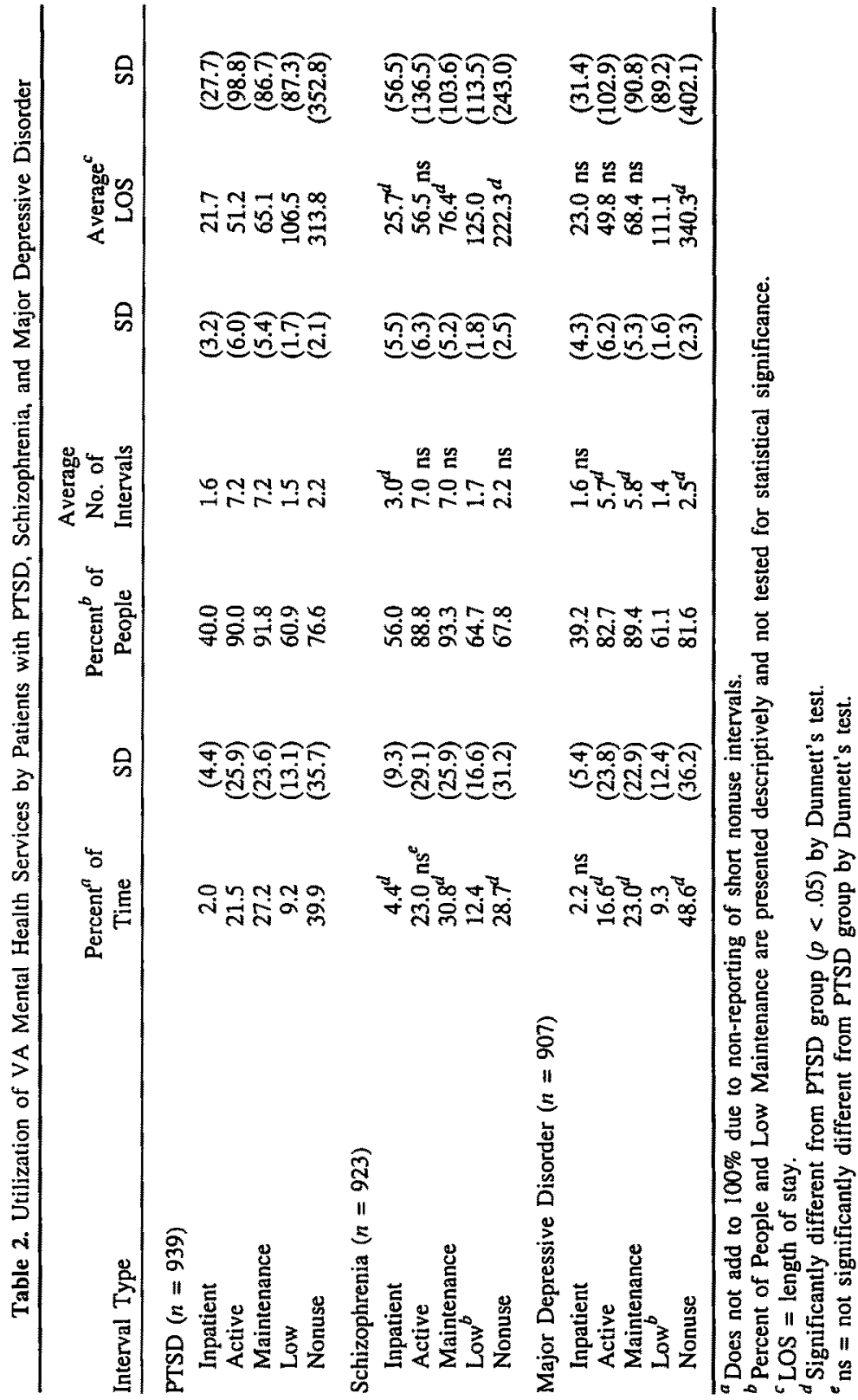


On average, PTSD patients spent $2 \%$ of their time (7.2 days per year) in inpatient mental health stays. Two out of five patients had at least one inpatient stay. The mean number of inpatient stays across all patients was 1.6. Patients who had at least one stay averaged 3.9 in the $43 / 4$ year study period. Thus there were large individual differences in use of inpatient services, with most patients using none, but with inpatient users averaging almost one hospitalization per year. The average LOS was 21.7 days.

\section{Comparison to Patients with Other Mental Disorders}

To put these patterns of utilization in perspective, patients with PTSD were compared to those with schizophrenia and major depressive disorder, also shown in Table 2. Based on the findings that active, maintenance, and nonuse accounted for most (89\%) of PTSD patients' time, and considering the resource intensive nature of inpatient care, these types of intervals were selected for comparative analyses. All utilization measures were positively skewed suggesting the desirability of a normalizing transformation (Neter, Wasserman, \& Kutner, 1990). Alternative transformations were tried and the $\log (x+1)$ transformation was usually most effective in reducing deviations from normality. For simplicity it was used on all variables. Statistical significance was tested by analysis of variance followed by Dunnett's test to compare patients with PTSD to each of the other groups.

Patients with PTSD spent less time in inpatient intervals (2\%) than did patients with schizophrenia (4\%), but did not differ from patients with major depressive disorder $(2 \%$; overall $F(2,2766)=47.89, p<.001)$. The average inpatient length of stay (LOS) was shorter for patients with PTSD (21.7 days) than for patients with schizophrenia (25.7), but did not differ from that of patients with major depressive disorder $(23.0$; overall $F(2,1246)=4.78$; $p=.009$ ). Patients with PTSD had fewer inpatient stays (1.6) than patients with schizophrenia (3.0), but did not differ from patients with major depressive disorder $(1.6$; overall $F(2,2766)=45.94, p<.001)$.

Use of outpatient mental health services also differed across the three diagnostic groups, but not in the same pattern as inpatient use. Patients with PTSD spent more time in active intervals (22\% of the time) than patients with major depressive disorder $(17 \%)$, but did not differ from patients with schizophrenia $(23 \%$; overall $F(2,2766)=21.82, p<.001)$. These findings were mainly due to the fact that patients with PTSD had more active intervals (7.2) than patients with major depressive disorder (5.7), but did not differ from patients with schizophrenia (7.0; overall $F(2,2766)=25.42$, $p<.001)$. The three diagnostic groups did not differ in their average LOS in active intervals (overall $F(2,2412)=2.66, p=.07$ ). 
PTSD patients spent more time in maintenance intervals $(27 \%$ of the time) than patients with major depressive disorder (23\%), and less time than patients with schizophrenia $(31 \%$; overall $F(2,2766)=25.49$; $p<.001$ ). This pattern was due both to differences in number of intervals and LOS. PTSD patients had significantly more maintenance intervals (7.2) than patients with major depressive disorder (5.8), but did not differ from patients with schizophrenia $(7.0$; overall $F(2,2766)=24.70$; $p<.001)$. Average LOS in maintenance intervals, on the other hand, was significantly shorter among patients with PTSD (65.1 days) than among patients with schizophrenia (76.4), but did not differ from that of patients with major depressive disorder $(68.4$; overall $F(2,2531)=14.72$; $p<.001)$.

Patients with PTSD were also intermediate between patients with the comparison diagnoses in the amount of time in nonuse intervals. Patients with major depressive disorder spent a higher proportion of their time in nonuse $(49 \%)$ than patients with PTSD $(40 \%)$, while patients with schizophrenia spent less time in such intervals $(29 \%)$ than patients with PTSD (overall $F(2,2766)=51.27, p<.001$ ). Patients with PTSD had fewer nonuse intervals (2.2) than patients with major depressive disorder (2.5), but did not differ from patients with schizophrenia (2.2; overall $F(2$, $2766)=8.88, p<.001)$. Patients with major depressive disorder had longer nonuse intervals (LOS $=340.3$ days) than patients with PTSD (313.8), while patients with schizophrenia had shorter such intervals (222.3 days; overall $F(2,2082)=56.38, p<.001)$.

Persistence. To examine persistence of use among the three diagnosis groups, we assessed utilization separately for each of the 4 years immediately following the 1986 outpatient mental health survey. Percent of time in each interval type was log-transformed and analyzed by repeated measures ANOVA, with diagnosis as the between-subjects factor and time (year) as the within-subject factor. The interaction of time with diagnosis was tested to see whether persistence of use differed among the three diagnostic groups.

There was an overall trend for decreasing inpatient use over time $(F(3,8298)=21.24, p<.001)$. Examination of the means revealed declines over the years in inpatient use by patients with PTSD and major depressive disorder but no decline for patients with schizophrenia. The interaction between diagnosis and time, however, was not significant $(F(6$, $8298)=1.92, p=.074$ ), apparently because of the high variance in inpatient use.

Time in outpatient active care also declined over successive years $(F(3,8298)=336.3, p<.001)$. There was a significant interaction between diagnosis and time $(F(6,8298)=12.18, p<.001)$ traceable to 
greater persistence in utilization by patients with schizophrenia. Time in outpatient maintenance also decreased over successive years $(F(3$, $8298)=185.2, p<.001)$. The rate of decline varied across diagnostic groups $(F(6,8298)=12.2, p<.001)$, with most persistence by patients with schizophrenia and least persistence by patients with major depressive disorder.

As might be expected given decreases in inpatient and outpatient utilization, time spent in nonuse intervals increased over successive years $(F(3,8298)=206.4, p<.001)$. A significant interaction between diagnosis and time indicated that the time trend differed for the three diagnostic groups $(F(6,8298)=4.01, p<.001)$. Time in nonuse increased more for patients with major depressive disorder and PTSD than for patients with schizophrenia. These changes in time in each interval type were paralleled by changes in the number of intervals of each type, but not by changes in length of stay.

To assess persistence of use at the individual level, we examined the year to year associations between the percentage of time in each interval type. There were substantial correlations across the years in time spent in each interval type. Though the strength of the associations dropped monotonically as the years being related were farther apart (average $r=.65$ for adjacent years, .51 for use 2 years apart) they were still notable (averaging .41) when use in the first year was correlated with use in the fourth year. The correlations were higher for active outpatient and nonuse intervals than for outpatient maintenance or inpatient intervals. This persistence in the pattern of use by individuals over time did not differ over the three diagnostic groups.

\section{Discussion}

Longitudinal analyses of use of VA mental health services by patients with PTSD over $43 / 4$ years were used to assess the episodic versus continuous nature of utilization, to examine the persistence of patterns of utilization over time, and to compare utilization by patients with PTSD to utilization by patients with two other serious mental disorders.

To summarize, the overall level of use by patients with PTSD was quite high. Nonuse accounted for a minority of their time. Patients with PTSD used inpatient services at a level comparable to patients with major depressive disorder and outpatient services at a level comparable to patients with schizophrenia. Conversely, patients with schizophrenia used more inpatient services and patients with major depressive disorder used less outpatient services than patients with PTSD. Taken together, over 
almost 5 years, patients with PTSD were treated with greater levels of mental health service than those used to care for patients with major depressive disorder, and less than those used to care for patients with schizophrenia.

Three out of four PTSD patients had at least one nonuse interval of greater than 100 days and most had more than one such interval. Those with a nonuse interval had an average of almost three, implying that most nonuse intervals were interruptions rather than terminations of use. On average, PTSD patients spent $40 \%$ of their time in nonuse intervals. So, use of VA mental health services by patients with PTSD was clearly episodic rather than continuous. Compared to patients with PTSD, patients with major depressive disorder spent more time in nonuse intervals and experienced more frequent and longer interruptions in care; patients with schizophrenia spent less time in nonuse and had shorter periods of nonuse. These findings are congruent with research indicating that the symptoms of schizophrenia are often episodic, while the symptoms of major depressive disorder are almost always episodic (Bleuler, 1978; Fenton \& McGlashan, 1991; Harding, 1988; Harding et al., 1987; Keller et al., 1992; Marengo et al., 1991; Shea et al., 1992; Stephens \& McHugh, 1991; Thase, 1990; Wells et al., 1992). It appears that PTSD may be intermediate between schizophrenia and major depressive disorder in the episodic nature of its course. To the extent that use of services reflects the course of symptoms of PTSD, these findings suggest that remissions are usually followed by relapse and do not indicate that the disorder has run its course.

The substantial correlations between patterns of use across several years indicates the existence of individual-level consistency in patterns across time and suggests that there might be distinct PTSD patient subgroups with different temporal patterns. One large apparent subgroup consists of episodic users. The finding that, for most patients with PTSD, utilization of mental health services is episodic raises questions about the goals and nature of treatment for PTSD. Within this population it may be much more realistic to focus on acute episodes with time-limited interventions seeking remission rather than using long-term intensive treatment. Recent promising results for brief behavioral treatments suggest that timelimited outpatient care can be helpful (Boudewyns \& Hyer, 1990; Cooper \& Clum, 1989; Solomon, Gerrity, \& Muff, 1992).

The finding that there was a second distinct subgroup of PTSD patients $(23 \%)$ who used mental health services continuously through the years of study, raises again the challenge of treatment matching. Continuous users might be responsive to outpatient treatment at fairly low frequency (outpatient maintenance in the terms of this analysis), perhaps 
combining psychopharmacological follow-up with relapse prevention and case management approaches (Davidson et al., 1990; Frank, Kosten, Giller, \& Dan, 1988; Solomon et al., 1992). Major challenges are to distinguish between these two groups of patients and to match the therapeutic approaches to the patients.

Correlations of PTSD patients' utilization in the first year with their utilization in the fourth year ranged from .31 to .50 indicating substantial individual-level stability of patterns of utilization and suggesting high persistence of use over the years. Very similar correlations were found for patients with schizophrenia and major depressive disorder, suggesting equivalent individual-level persistence or stability in the three diagnostic groups.

Both inpatient and outpatient use showed declines over four years of observation. For example, patients with PTSD, who used about 10 days of inpatient care in the first year, used about 6 days in the fourth year of follow-up, a decline of $41 \%$ over these years. Similarly, these patients spent $30 \%$ of the first year in active outpatient intervals and $18 \%$ of the fourth year in such intervals. Decreases in use by patients with PTSD were intermediate between those of patients with major depressive disorder and schizophrenia, with a more rapid decline apparent among patients with major depressive disorder and a slower decline among those with schizophrenia.

On the face of it these results suggest moderate persistence in use of mental health services by patients with PTSD with a half-life of about 5 years. Such a projection, however, does not consider either the episodic nature of PTSD or the statistical process of regression toward the mean in samples selected based on their utilization at one time. The selection process for this study increased the chance that utilization would appear to decrease over time, whether or not utilization decreases among all veterans with PTSD. That is, patients were selected for study because they used outpatient mental health services in the fall of 1986. By virtue of selection, the patients had to be in an episode of mental health service utilization, and on average, their outpatient use at time of selection could be expected to be biased upward compared to their own typical level of use. Thus, because of the selection process, use would likely appear to decrease over time by the process of regression to the mean - with or without any decrease in use among unselected patients. To assess the potential effects of regression to the mean, a subset of the sampled patients were selected who were low users of services ( 0 or 1 visits, no inpatient days) during the first year after the outpatient survey. Among these patients, utilization increased dramatically from year 1 to year 2 , and continued to increase in each successive year. For example, time in 
active outpatient intervals increased from $0.03 \%$ to $1.68 \%$, to $1.90 \%$, to $2.30 \%$. Thus, regression to the mean must be at least partially responsible for the decreasing trends observed in this study and in other studies in which patient selection is dependent on utilization (or symptoms) (Beebe, 1988).

The decline in use over 4 years among patients with PTSD was less than the decline among patients with major depressive disorder but more than that among patients with schizophrenia. The differences among the groups may simply reflect the different means toward which the three groups are regressing, rather than differential persistence per se. Correlations of utilization across years were very similar for the three diagnostic groups, suggesting that actual persistence may be similar for patients with these disorders.

Given the apparently episodic nature of use by patients with PTSD, any method of assessing persistence of PTSD that concludes that the condition is cured or in permanent remission based on the absence of utilization or symptoms for periods as long as a year is likely to be seriously biased, since utilization, and presumably symptoms, appear very likely to return in subsequent years. Thus, use of services by patients with PTSD is substantially more persistent than suggested by simple interpretation of the current time trends or by results showing prevalence of PTSD after a delay.

The major limitation of this study was that we looked only at utilization. The level, continuity, and persistence of utilization are of interest in themselves, but we would also like to draw inferences about the progression of symptoms and the underlying disorders. To assess the natural history of the disorders more directly, one needs longitudinal data on the symptomatology itself. Such data are extremely difficult and costly to obtain. The current results provide direct information about the time course of utilization of VA mental health services by patients with PTSD and are suggestive about the time course of the condition.

A second important limitation results from the method of diagnosis and selection of patients. Patients were selected based only on clinical diagnoses in the outpatient setting, without further validation. Given the low number of disorders per patient reported in the outpatient survey, and the low rate of major depressive disorder diagnoses among patients with PTSD, it is likely that diagnoses were underreported and that the three groups selected in the study were far from pure, including some patients who might not meet all the criteria for the reported diagnosis, and some patients who had more than one of the three disorders. In particular, it is likely that the PTSD group includes some patients with major depressive disorder, with or without symptoms at the time of the outpatient survey. In addition, because information 
about the specific purpose of visits was generally unavailable, the utilization classification system simply included all VA mental health care. So the data on use of mental health care by patients with each disorder undoubtedly included some care that was primarily for other mental health problems, including some care for the comparison conditions. The combined result of these diagnostic limitations were that (a) the amount of use by patients in any of the three diagnostic groups overestimated the amount of use they made for that particular disorder, and (b) differences found among the three groups probably underestimated the differences among pure diagnostic groups. These biases do not threaten the major conclusions of the study that use of mental health services by patients with PTSD is episodic, persistent, and substantial (being between the levels of use by patients with schizophrenia and major depressive disorder).

Another limitation of the study was that we examined only utilization of VA mental health services, while patients may have used non-VA services and may have received mental health care in nonmental health settings within the VA. This is potentially important, since an extended period using non-VA mental health care in lieu of VA care would be misinterpreted as a period of nonuse and inappropriately form the basis for concluding that use is episodic. This is unlikely to be a significant problem in the current study, since patients were selected based on their use of VA mental health services. Fewer than $30 \%$ of VA mental health care users reported any use of non-VA physical or mental health services in 6 months (Ronis, Bates, \& Wolff, 1992a). Moreover, in order to incorrectly conclude that a patient was an episodic user, he or she had to switch to non-VA use and then switch back. On average, PTSD patients with at least one nonuse interval had close to three of them. Given the low use of non-VA services, it seems highly unlikely that our estimate of episodic use, as indicated by nonuse intervals, was notably biased.

Generalizability of these results to all persons with these disorders is limited by our attempt to exclude patients who had more than one of the three disorders and the restriction to veterans using VA services. Studies have found that from $26 \%$ to $84 \%$ of patients with PTSD have a lifetime comorbidity of major depressive disorder (Keane \& Wolfe, 1990). The PTSD treated within the VA is almost all combat-related and VA users are a largely male, low income group, with military experience, and a bimodal age distribution corresponding to two major military mobilizations: World War II and the Vietnam war. The race distribution among these patients is unknown. Future studies should extend the examination of temporal patterns of PTSD to other samples, including more females, more patients in other age groups, and patients with noncombat-related PTSD, and should determine the racial and ethnic characteristics of the samples. 
In sum, PTSD patients made extensive use of inpatient and outpatient mental health services. Though utilization declined over the 4 years of the study, at least part of the decline was due to regression to the mean. Most PTSD patients had more than one interruption in mental health service utilization, each lasting more than 100 days, indicating that use was episodic rather than continuous. To the extent that use of VA mental health services reflects the course of the disorder, this implies that remissions are usually followed by relapse, and that absence of symptoms does not mean that the disorder has run its course. In general, the intensity, persistence, and continuity of mental health service use among PTSD patients were higher than among patients with major depressive disorder, but not as high as among patients with schizophrenia.

\section{Acknowledgments}

We thank Matthew J. Friedman, Patrick Boudewyns, and John L. Harrington, for their comments on a preliminary version of the classification system, and Donald M. Steinwachs, for comments on an earlier draft of the paper. This research was supported by the Chief Medical Director's Special Committee on PTSD, Department of Veterans Affairs.

\section{References}

American Psychiatric Association (1980). Diagnostic and statistical manual of mental disorders (3rd ed). Washington DC: Author.

Beebe, J. C. (1988). Medicare reimbursement and regression to the mean. Health Care Financing Review, 9, 9-22.

Bleuler, M. (1978). The schizophrenic disorders: The long term patient and family studies. Translated by S. M. Clemens. New Haven: Yale University Press.

Boudewyns, P. A., \& Hyer, L. (1990). Physiological response to combat memories and preliminary treatment outcome in Vietnam veteran PTSD patients treated with direct therapeutic exposure. Behavior Therapy, 21, 63-87.

Burns, B. J., Wagner, H. R., \& Taube, J. E. (1993). Mental health service use by the elderly in nursing homes. American Joumal of Public Health, 83, 331-337.

Cooper, N. A., \& Clum, G. A. (1989). Imaginal flooding as a supplementary treatment for PTSD in combat veterans: A controlled study. Behavior Therapy, 20, 381-391.

Davidson, J., Kudler, H., Smith, R., Mahorney, S. L., Lipper, S., Hammett, E., Saunders, W. B., \& Cavenar, Jr., J. O. (1990). Treatment of posttraumatic stress disorder with amitriptyline and placebo. Archives of General Psychiatry, 47, 259-266.

Fenton, W. S., \& McGlashan, T. H. (1991). Natural history of schizophrenia subtypes: II. Positive and negative symptoms and long-term course. Archives of General Psychiatry, 48, 978-986.

Frank, J. B., Kosten, T. R., Giller, Jr., E. L., \& Dan, E. (1988). A randomized clinical trial of phenelzine and imipramine for posttraumatic stress disorder. American Joumal of Psychiatry, 145(10), 1289-1291.

Green, B. L., Lindy, J. D., \& Grace, M. C. (1985). Posttraumatic stress disorder: Toward DSM-IV. Joumal of Nervous and Mental Disease, 173, 406-411. 
Green, B. L., Lindy, J. D., Grace, M. C., \& Leonard, A. C. (1992). Chronic posttraumatic stress disorder and diagnostic comorbidity in a disaster sample. Joumal of Nervous and Mental Disease, 180, 760-766.

Harding, C. M. (1988). Course types in schizophrenia: An analysis of European and American studies. Schizophrenia Bulletin, 14, 633-643.

Harding, C. M., Brooks, G. W., Ashikaga, T., Strauss, J. S., \& Breier, A. (1987). The Vermont longitudinal study: II. Long-term outcome for subjects who retrospectively met DSM-III criteria for schizophrenia. American Journal of Psychiatry, 144, 727-735.

Hornbrook, M. C., Hurtado, A. V., \& Johnson, R. E. (1985). Health care episodes: definition, measurement and use. Medical Care Review, 42, 163-218.

Horowitz, M. J. (1986). Stress response syndromes (2nd ed.). Northvale, NJ: Jason Aronson.

Keane, T. M., \& Wolfe, J. (1990). Comorbidity in post-traumatic stress disorder: An analysis of community and clinical studies. Joumal of Applied Social Psychology, 20(21), 1776-1788.

Keeler, E. B., Manning, W. G., \& Wells, K. B. (1988). The demand for episodes of mental health services. Journal of Health Economics, 7, 369-392.

Keller, M. B., Lavori, P. W., Mueller, T. I., Endicott, J., Coryell, W., Hirschfeld, R. M. A., \& Shea, T. (1992). Time to recovery, chronicity, and levels of psychopathology in major depression: A 5-year prospective follow-up of 431 subjects. Archives of General Psychiairy, 49, 809-816.

Kessler, L. G., Steinwachs, D. M., \& Hankin, J. R. (1980). Episodes of psychiatric utilization. Medical Care, 18, 1219-1227.

Kinzie, J. D. (1986). Severe posttraumatic stress syndrome among Cambodian refugees: Symptoms, clinical course, and treatment approaches. In J. H. Shore (Ed.), Disaster stress studies: New methods and findings (pp. 124-140). Washington D.C.: American Psychiatric Press.

Kulka, R. A., Schlenger, W. E., Fairbank, J. A., Hough, R. L., Jordan, B. K., Marmar, C. R., \& Weiss, D. S. (1990a). Trauma and the Vietnam War generation: Repont of findings from the National Vietnam Veterans Readjustment Study. New York: Brunner/Mazel.

Kulka, R. A., Schlenger, W. E., Fairbank, J. A., Hough, R. L., Jordan, B. K., Marmar, C. R., \& Weiss, D. S. (1990b). The National Vietnam Veterans Readjustment Study: Tables of findings and technical appendices. New York: Brunner/Mazel.

Marengo, J., Harrow, M., Sands, J., \& Galloway, C. (1991). European versus U.S. data on the course of schizophrenia. American Joumal of Psychiatry, 148, 606-611.

Neter, J., Wasserman, W., \& Kutner, M. H. (1990). Applied linear statistical models: Regression, analysis of variance, and experimental designs (3rd ed). Boston: Inwin.

Ronis, D. L., Bates, E. W., \& Wolff, N. (1992a). 1990 Survey of Outpatient Mental Health and Readjustment Counseling Services: Final Descriptive Findings, August 1992. Working Paper WP92-017. Available from HSR\&D Field Program (152), P.O. Box 130170, Ann Arbor, MI 48113-0170, (313) 930-5100.

Ronis, D. L., Bates, E. W., Wolff, N. (1992b). 1990 Survey of Outpatient Mental Health and Readjustment Counseling Services: Analyses of Diagnoses and Problems, August 1992. Working Paper WP92-016. Available from HSR\&D Field Program (152), P.O. Box 130170, Ann Arbor, MI 48113-0170, (313) 930-5100.

Sack, W. H., Clarke, G., Him, C., Dickason, D., Goff, B., Lanham, K., \& Kinzie, J. D. (1993). A 6-year follow-up study of Cambodian refugee adolescents traumatized as children. Joumal of the American Academy of Child and Adolescent Psychiatry, 32, 431-437.

Shea, M. T., Elkin, I., Imber, S. D., Sotsky, S. M., Watkins, J. T., Collins, J. F., Pilkonis, P. A., Beckham, E., Glass, D. R., Dolan, R. T., \& Parloff, M. B. (1992). Course of depressive symptoms over follow-up: Findings from the National Institute of Mental Health Treatment of Depression Collaborative Research Program. Archives of General Psychiatry, 49, $782-787$.

Solomon, S. D., Gerrity, E. T., \& Muff, A. M. (1992). Efficacy of treatments for posttraumatic stress disorder: An empirical review. Journal of the American Medical Association, 268(5), 633-638.

Solomon, Z., Kotler, M., Shalev, A., \& Lin, R. (1989). Delayed onset PTSD among Israeli veterans of the 1982 Lebanon War. Psychiatry, 52, 428-436. 
Stephens, J. H., \& McHugh, P. R. (1991). Characteristics and long-term follow-up of patients hospitalized for mood disorders in the Phipps Clinic, 1913-1940. Joumal of Nervous and Mental Disease, 179, 64-73.

Thase, M. E. (1990). Relapse and recurrence in unipolar major depression: Short-term and long-term approaches. Joumal of Clinical Psychiatry, 51[6 (Suppl)], 51-57.

Weiss, D. S., Marmar, C. R., Schlenger, W. E., Fairbank, J. A., Jordan, B. K., Hough, R. L., \& Kulka, R. A. (1992). The prevalence of lifetime and partial post-traumatic stress disorder in Vietnam theater veterans. Journal of Traumatic Stress, 5, 365-376.

Wells, K. B., Burnam, M. A., Rogers, W., Hays, R., \& Camp, P. (1992). The course of depression in adult outpatients: Results from the Medical Outcomes Study. Archives of General Psychiatry, 49, 788-794. 